Titanic had created a media storm. A videotape of the wreck, handed up to an opportunist long-range helicopter and addressed rather pompously "To the World Press", had been quickly broadcast as a "CBS exclusive" - a silly business that predictably upset Ballard's French partners in the expedition.

Knowing some of his scientific papers, I judge that there is not enough Ballard (or too much Hively) in this book. There is also some thoughtless editing, especially in the figures — some need scales, or are over-reduced or have unhelpful legends, while a few are downright misleading. Oddly, there are 60 pages of "further reading", simply listing references to the primary literature, although not one is cited in the text. Perhaps they are the contents of Ballard's reprint boxes, given to his ghost-writer for background, and arranged sequentially by year?

Alan Longhurst is at Place de l'Eglise, 46160

Cajarc, France.

\section{Winging their way}

The Biomechanics of Insect Flight: Form, Function, Evolution

by Robert Dudley

Princeton University Press: 2000. 476 pp.

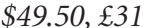

\section{Form and Function of Insect}

Wings: The Evolution of Biological Structures

by Dmitry L. Grodnitsky

Johns Hopkins University Press: 1999. 261 pp. $\$ 49.95, £ 41.50$

\section{R. McNeill Alexander}

Most animal species are insects, and the most conspicuous characteristic of the insects is that they fly. Research on insect flight has been held back by approaches that, with hindsight, seem crude and inappropriate, but the past two decades have brought many exciting advances.

Previously, we thought of insect wings as stiff, flat plates - now we know that some bend and twist in flight in ways that must have large aerodynamic effects. It used to seem prohibitively difficult to make physiological measurements on free-flying insects, or even to obtain film of adequate quality for detailed analysis of the movements of free flight, so most observations were made on insects induced to flap their wings while glued to supporting wires. Now we can measure the oxygen consumption of a single bee flying free in a wind tunnel, and even more ambitious experiments are in prospect.

Another approach now seen to have limited value is the use of blade-element theory, which works for helicopter rotors, to estimate aerodynamic forces on insect wings. Calculations give the maximum forces the wing motion should be able to generate. In many cases, these are much less than is needed to keep the animal airborne, inspiring the dictum that engineers have proved bumblebees cannot fly. Bumblebees have, of course, convincingly proved otherwise.

It has long been realized that the crucial difference is that aircraft aerofoils move more or less steadily through the air, whereas the flapping wings of insects repeatedly reverse their direction of motion, giving rise to effects that are not predicted by 'steadystate' aerodynamics. Blade-element theory can do no more than tell us when steadystate aerodynamics is inadequate; even when it shows that steady-state forces could support the insect, it cannot tell us that no unsteady effects are acting, and it can never tell us what the unsteady effects are. The focus has recently shifted from the movements of the wings to the movements of the air they drive, leading to qualitative explanations of aerodynamic mechanisms that will soon, one hopes, become quantitative.

Much less progress has been made in the possible energy-saving role of elastic structures. It has long been known that some insects could save a lot of energy if the kinetic energy taken from the wings at the end of each stroke could be stored and returned in an elastic recoil. Arguments that this does or does not happen have generally depended on estimates of the efficiency with which the flight muscles must work. If the assumption of no elastic storage led to implausibly high

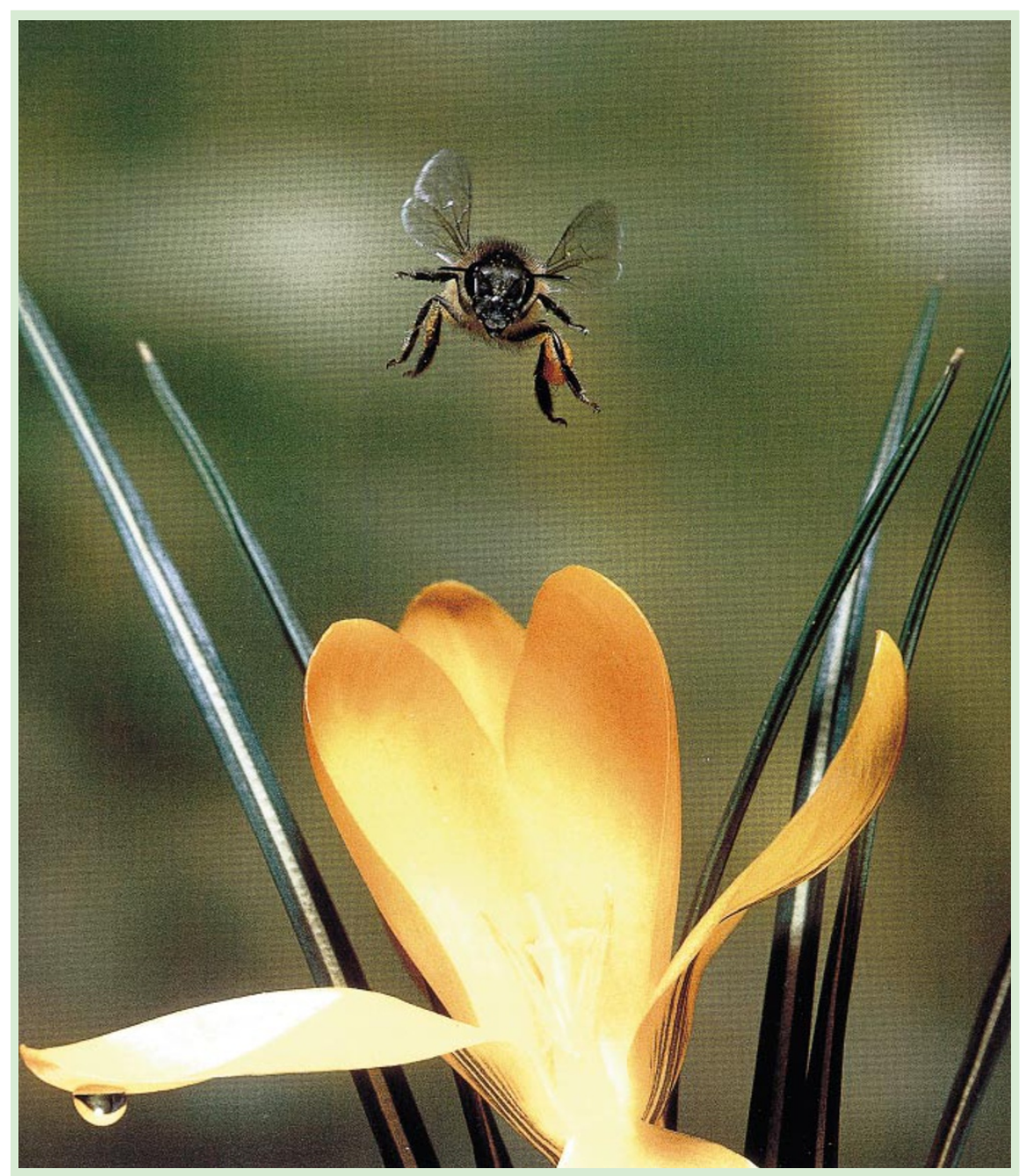

\title{
Flight recordings
}

One of photographer Stephen Dalton's first assignments, in the 1960s, was a book about honeybees. His discovery that insect flight had never been clearly caught in a still photo launched both an obsession with solving this problem and an award-winning career. The results, building on recent advances in highspeed photography, are a highlight of his latest book Secret Worlds (Firefly, \$35). In 125 colour photos, each with explanatory text, some animals are seen hiding or resting. But Dalton's fascination with capturing movement creates the most memorable images: the agility of a diving vole, a cockchafer coming in to land, the aerial manoeuvres of bats, and the astonishing sight of a basilisk running across water. 
estimates of efficiency, it was concluded that elastic mechanisms must operate. Unfortunately, it has not been clear how high an efficiency is plausible, and there have been no satisfactory calculations of energy savings based on measurements of the elastic properties of the thorax. An inspired new technique is needed to advance our knowledge, but 'work loop' experiments have made important steps in the right direction. In these muscles are stimulated while being stretched and allowed to shorten at rates that simulate the action of flight.

Robert Dudley has written a remarkably comprehensive account of our knowledge of insect flight: his bibliography fills 102 pages. No other recent book covers the field so fully, so this one will be most valuable to students and researchers. But his aim seems to have been to inform rather than to teach. The book reads like a series of review articles on the morphology, evolution, aerodynamics, physiology and control of flight. There are remarkably few illustrations and too little mathematics. There is frustratingly little detail about the methods and results of even the most significant experiments. However, he has tried to help by providing chapter summaries, a list of symbols and a glossary.

Dmitry Grodnitsky has written a book of much narrower scope, with far more experimental and morphological detail. It starts with the experiments in which he and his colleagues visualized the air movements in the wake of tethered insects beating their wings in still air. Observations on free flight would have been preferable, had they been feasible, but these demonstrations of vortex structure are nevertheless valuable. The book continues with discussions of the evolution of insect flight, of the functional morphology of the veins and folds of insect wings, and of the scales on the wings of butterflies and moths. This is a book for specialists.

R. McNeill Alexander is in the School of Biology, University of Leeds, Leeds LS2 9JT, UK.

\section{Strolling through the bioenergy field}

\section{The Energy of Life \\ by Guy Brown \\ Free Press: 2000.288 pp. \$25, £16.99}

\section{Paul D. Boyer}

Guy Brown defines energy loosely. "We have many words to express the high energy state: vitality, vigor, vivacity, strength, arousal, ardor, drive, fervor, stamina, gumption, zeal and zest," he states. Such a definition allows the author to explore the basis of many aspects of human behaviour in this unusual and readable book.

Brown is a respected researcher in the field of bioenergetics and is well served by his broad knowledge and historical perspective. His introductory descriptions of how living processes are made possible by the wondrous machinery of the cell are what I would like to give my non-scientific friends who ask what my work is about. Avoiding technical details, he describes such processes as how DNA is a blueprint for some 50,000 proteins that make possible the occurrence, control and complexity of metabolic pathways, and that energy is captured in the structure of a molecule called ATP.

In a chapter on "The body electric", however, he appears over-enthusiastic in the use of the term 'electricity', including not only the transfer of electrons, but also the use of proton and sodium gradients as proton and sodium electricity and the use of ATP as phosphate electricity. Most bioenergeticists would feel the statement "Our cells are energized by huge electric fields driving currents of charged particles via a myriad minuscule wires" does not convey a proper perspective.

Brown is writing for a general audience, though even graduate students in biochemistry would enjoy his historical accounts of the development of current understanding in such areas as glycolysis, the electrontransport chain, the capture of energy by development of electrochemical proton gradients, and the use of phosphate-bond energy. Other nuggets scattered throughout the book will interest and entertain readers in the fields of bioenergetics and biochemistry.

Mitochondria, the cellular organelles in which ATP is made, were introduced into our cells billions of years ago by fusion with a microorganism. It is from these organelles that free radicals escape and damage our DNA and proteins, eventually leading to ageing. As Brown emphasizes, a wandering lone electron may wreak havoc with hundreds of molecules. Although antioxidants do play a preventive role, he inappropriately implies that purified vitamins $\mathrm{C}$ and $\mathrm{E}$ and beta-carotene are less effective than natural antioxidants. It is the structure, not a 'natural' source, that is important. The tendency of mitochondria to leak protons and radicals is regarded as a fault in nature's design. But another view is that they are a splendidly honed organelle that captures most of the energy released by use of toxic oxygen, with enough preventive and repair mechanisms to allow us to reach old age.

Brown ranges widely, from motion to metabolic rate and on to obesity, including chapters on mind energy, brain waves, and sex and sleep. He recognizes that social, psychological and neurophysiological factors all play a role in the pace of life. (Ilearned that giving rats a shot of adrenaline right after a learning experience increases memory retention.) We can all relate his good discussion of the effect of stress on metabolism and behaviour to our own experiences.
We learn that Sigmund Freud was not a well man (he suffered from chronic fatigue and nervousness, and was enthusiastic about cocaine); that the term 'mesmerism' was derived from Viennese physician Franz Mesmer, who attributed powers to a force called animal magnetism; that Benjamin Franklin headed a commission which concluded that such a force did not exist; and that for better sex one should try the daytime or early evening. In the chapter on sex and sleep, Brown even discusses life in the scientific community. Discoveries such as that of nitric oxide as a signal transmitter create a "wave of interest in the scientific community"; if you succeed in getting on to the wave of discovery, your career starts to flourish.

A 58-page appendix, "The story of living energy", does not meet the objective stated in the introduction as giving the background ideas in bioenergetics. Instead, it is an elegant historical account of how an understanding of life's processes has been acquired, from the insights of ancient Greece to the recognition that fermentation is a function of living cells. Readers are exposed to such novelties as the type of healthy regime Socrates might have prescribed.

The very wide range of topics Brown considers, and his slight tendency to wander and repeat himself, do not detract importantly from this informative and enjoyable account of the scientific basis of our everyday life and behaviour.

Paul D. Boyer is in the Department of Chemistry and Biochemistry, University of California,

Los Angeles, California 90095, USA.

\section{New in paperback}

The Unnatural Nature of Science

by Lewis Wolpert

Faber \& Faber, £8.99

Living with our Genes: Why They Matter More Than You Think

by Dean Hamer \& Peter Copeland Pan, £6.99

Hermaphrodites and the Medical Invention of Sex

by Alice Domurat Dreger

Harvard University Press, $£ 10.50$, \$16.95

"Ignore the somewhat trendy title, which might seem to threaten yet another piece of pretentious postmodernism. This is a well-researched, sober history of a problem that Alice Dreger shows has directly affected more people than we might think and which shapes the sense of sexual identity of us all ... Dreger shows how deeply ingrained are our assumptions about gender normality (sexual anatomy is destiny), and on how flimsy a basis they have been grounded. The book offers us all a lesson in self-awareness." Roy Porter, Nature 393, 323 (1998) 